\title{
Advantages and disadvantages of using Carbon Nanostructures in Reproductive Medicine: two sides of the same coin
}

\author{
Hadi Zare-Zardini ${ }^{1,2,3}$, Nooshin Hatamizadeh ${ }^{4}$, Navid Haddadzadegan ${ }^{5}$, Hossein Soltaninejad ${ }^{6}$, Mojgan Karimi- \\ Zarchi $^{7}$ \\ ${ }^{1}$ Hematology and Oncology Research Center, Shahid Sadoughi University of Medical Sciences, Yazd, Iran \\ 2Medical Nanotechnology and Tissue Engineering Research Center, Yazd Reproductive Sciences Institute, Shahid \\ Sadoughi University of Medical Sciences, Yazd, Iran \\ ${ }^{3}$ Department of Biomedical Engineering, Meybod University, Meybod, Iran \\ ${ }^{4}$ Department of Obstetrics and Gynecology, Shahid Sadoughi University of Medical Sciences, Yazd, Iran \\ ${ }^{5}$ Department of Anesthesiology and Critical Care, Shahid Sadoughi University of Medical Sciences, Yazd, Iran \\ ${ }^{6}$ Department of Nanobiotechnology, Faculty of Biological Sciences, Tarbiat Modares University, Tehran, Iran \\ ${ }^{7}$ Department of Gynecology Oncology, Firoozgar Hospital, Iran University of Medical Sciences, Tehran, Iran
}

\begin{abstract}
Carbon nanostructures are important nanomaterial with interesting physical and chemical properties. These nanostructures have been assessed for application in different fields of medicine, such as cancer detection and treatment, Parkinson disease, reproductive medicine, etc. This nanomaterial can be used in reproductive medicine as a drug delivery system, antifungal, antiviral, and antibacterial agent, condom-coating agent, enhancer of sperm fertilizing ability, ectopic pregnancy treatment, trophoblastic diseases, endometriosis, uterine fibroids, and Assisted Reproduction Techniques (ART) improvement. The other side of this coin involves various side effects of carbon nanostructures, especially negative effects on reproductive systems. All carbon nanostructures showed toxicity on the reproductive system by producing reactive oxygen species and oxidative stress. Less attention has been given to the unique properties of carbon nanostructures, except for their practical attractiveness, the other side of this coin, namely the risks and side effects of these compounds - especially in the case of a reproductive system that supports the survival and health of future generations. Therefore, we suggest paying particular attention to the negative aspects of the increasing use of carbon nanostructures.
\end{abstract}

Keywords: carbon nanostructures, reproductive medicine, side effects, attention

\section{BACKGROUND}

Application of different nanostructures (nanocages, magnetic nanochains, nanocomposites, nanofabrics, nanofibers, nanoflowers, nanofoams, nanoholes, nanomesh, nanopillars, nanopin film, nanoplatelet, nanoribbon, nanoring, nanorod, nanosheet, nanoshell, nanotip, nanowires, nanostructured film, quantum dot, lipid nanostructures, and etc.) has been developed in different fields over the past years, especially in medicine (Zahid et al., 2013; ZareZardini et al., 2013; 2015; 2016; 2018a; 2018b; 2020). Carbon nanostructures with various shapes (graphene and its derivatives, SWCNTs, MWCNTS, and fullerene) have amazing physical and chemical properties including thermal and electrical conductivity, vibroelectronic properties, high aspect ratio, strength and elasticity, electron emission, high tensile strength, high flexibility, and etc. so, due to these properties, carbon nanostructures can be used in the field of medicine (Jeevanandam et al., 2018; Mukhin et al., 2015; Patel et al., 2020).

One side of the coin, e.g. the use of carbon nanostructures in reproductive medicine, has been the subject of many studies. Effective and positive mechanisms of these nanostructures in various fields of reproductive medicine, such as increasing sperm fertility, prevention of unplanned pregnancy, increasing sperm and egg retention in culture medium, preventing the transmission of sexually transmitted infections, increasing the effectiveness of genital health drugs such as chemotherapy in the treatment of reproductive system cancers, and etc., has been well studied and proven, and the most detailed topics related to these positive effects have been discussed in many papers The use of carbon nanostructures in ART include delivery system for increase bioavailability and permeability of sexual hormones, gametes and embryos selection, and gene transfer. In the field of embryogenesis, these nanostructures can be used for labelling pre-implanted embryos, Nano nutrition, and gene therapy. Carbon nanostructures have been used in reproductive oncology for treatment and diagnostic purposes. Infection detection, targeted treatment, microbicides and vaccine design for prevention, are all applications of Carbon nanostructures in the field of reproductive infections. These nanostructures can be used as alternative for surgical intervention in ectopic pregnancy, trophoblastic diseases, endometriosis and uterine fibroids (Azizi-Lalabadi et al., 2020; Bernabò et al., 2019; 2020; George et al., 2018; He et al., 2013; Kang et al., 2007; Krishnaraj et al., 2014; Liu et al., 2017; Liu \& Chen, 2016; Maleki Dizaj et al., 2015; Ramal-Sanchez et al., 2019; Saliev, 2019; Zare-Zardini et al., 2020).

However, attention to the other side of this coin, namely the risks for increasing the use and the range of carbon nanostructures, especially their toxicity on the reproductive system, has been neglected. Compared to the positive aspects of the use of carbon nanostructures in reproductive medicine, much less attention has been paid to its negative aspects ( $\mathrm{Za}$ re-Zardini et al., 2018a; 2018b) (Figure 1). A comparison of the number of published papers in both fields and the significant differences in these areas prove this claim. Carbon nanostructures have different side effects on normal cells, tissues and organs. All shapes of these nanostructures showed adverse toxicity on reproductive systems. Among carbon nanostructures, it has been proven that the highest side effects belong to SWCNTs. The lowest toxicity was seen in the application of Fullerene. Functionalization of graphene with active 


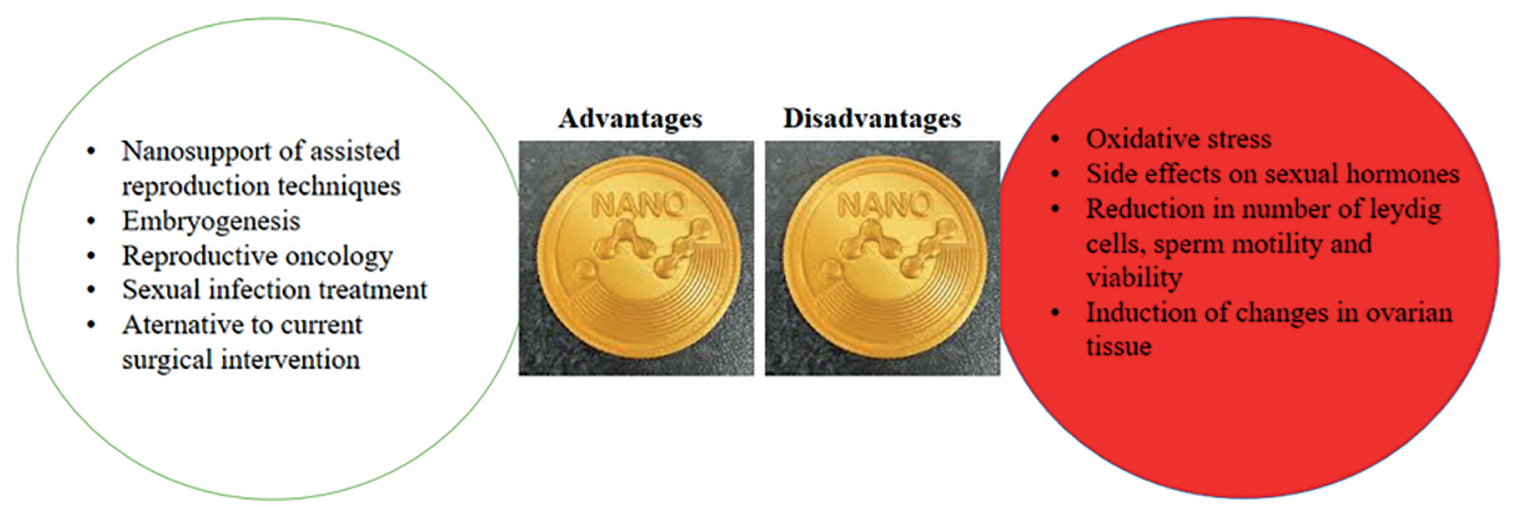

Figure 1. The two sides of the coin for using carbon nanostructures in reproductive medicine.

functional groups such as $-\mathrm{COOH}$ and $-\mathrm{NH} 2$ lead to increased toxicity on the reproductive system. Reduced graphene had the lowest toxicity among all types of graphene. There are limited studies on the mode of negative impacts of these nanostructures on the reproductive system, but these limited studies have shown that the main acceptable mechanism in the toxicity of carbon nanostructures on the reproductive system is the production of reactive oxygen species and oxidative stress, side effects on sexual hormones, reduction in the number of Leydig cells, sperm motility and viability, and induction of changes in ovarian tissue, such as change in the expression of genes involved in ovarian hormones and cytokine pathways, disruption of balanced hormone levels by effect on hypothalamic-pituitary-gonadal axis or stimulation of secretory cells, reduction of steroidogenesis in the ovary, and so on. The negative effects of carbon nanostructures on the male reproductive system are greater than on the female reproductive system due to lack of suitable protection mechanisms for the testes (Asghar et al., 2016; Dehghanbaghi \& Zare-Zardini, 2019; Xu et al., 2016; Vasyukova et al., 2015).

Due to less attention to the negative aspects regarding the use of carbon nanostructures, we suggest that in future studies and research, more attention be paid to other aspects, namely the harmful effects related to carbon nanostructures. Due to the importance of reproductive system health for survivors and the health of born children, it is highly important to pay attention to the negative impact of carbon nanostructures on the reproductive system.

\section{CONFLICT OF INTEREST}

The authors have no conflict of interest to declare.

\section{Corresponding authors:}

Hadi Zare-Zardini

Hematology and Oncology Research Center

Shahid Sadoughi Hospital

Shahid Sadoughi University of Medical Sciences

Yazd, Iran

E-mail: hadizarezardini@gmail.com

\section{REFERENCES}

Asghar W, Shafiee H, Velasco V, Sah VR, Guo S, El Assal $R$, Inci $F$, Rajagopalan $A$, Jahangir $M$, Anchan RM, Mutter GL, Ozkan M, Ozkan CS, Demirci U. Toxicology Study of Single-walled Carbon Nanotubes and Reduced Graphene Oxide in Human Sperm. Sci Rep. 2016;6:30270. PMID: 27538480 DOI: $10.1038 /$ srep30270
Azizi-Lalabadi M, Hashemi H, Feng J, Jafari SM. Carbon nanomaterials against pathogens; the antimicrobial activity of carbon nanotubes, graphene/graphene oxide, fullerenes, and their nanocomposites. Adv Colloid Interface Sci. 2020;284:102250. PMID: 32966964 DOI: 10.1016/j. cis. 2020.102250

Bernabò N, Machado-Simoes J, Valbonetti L, Ramal-Sanchez M, Capacchietti G, Fontana A, Zappacosta R, Palestini P, Botto L, Marchisio M, Lanuti P, Ciulla M, Di Stefano A, Fioroni E, Spina M, Barboni B. Graphene Oxide increases mammalian spermatozoa fertilizing ability by extracting cholesterol from their membranes and promoting capacitation. Sci Rep. 2019;9:8155. PMID: 31148593 DOI: $10.1038 / \mathrm{s} 41598-019-44702-5$

Bernabò N, Valbonetti L, Raspa M, Fontana A, Palestini $P$, Botto $L$, Paoletti R, Fray $M$, Allen $S$, Machado-Simoes J, Ramal-Sanchez M, Pilato S, Scavizzi F, Barboni B. Graphene Oxide Improves in vitro Fertilization in Mice With No Impact on Embryo Development and Preserves the Membrane Microdomains Architecture. Front Bioeng Biotechnol. 2020;8:629. PMID: 32612987 DOI: 10.3389/ fbioe. 2020.00629

Dehghanbaghi $\mathrm{N}$, Zare-Zardini $\mathrm{H}$. Investigating the toxicity of carbon nanostructures on reproductive systems: A systematic review. In: The 8th International Congress and Student Festival of Reproductive Medicine; 2019 Apr 19; Yazd, Iran. Avaiable at: https://civilica.com/doc/912287/

George G, Sisupal SB, Tomy T, Kumaran A, Vadivelu P, Suvekbala V, Sivaram S, Ragupathy L. Facile, environmentally benign and scalable approach to produce pristine few layers graphene suitable for preparing biocompatible polymer nanocomposites. Sci Rep. 2018;8:11228. PMID: 30046158 DOI: $10.1038 / s 41598-018-28560-1$

He H, Pham-Huy LA, Dramou P, Xiao D, Zuo P, PhamHuy C. Carbon nanotubes: applications in pharmacy and medicine. Biomed Res Int. 2013;2013:578290. PMID: 24195076 DOI: $10.1155 / 2013 / 578290$

Jeevanandam J, Barhoum A, Chan YS, Dufresne A, Danquah MK. Review on nanoparticles and nanostructured materials: history, sources, toxicity and regulations. Beilstein J Nanotechnol. 2018;9:1050-74. PMID: 29719757 DOI: 10.3762/bjnano.9.98 
Kang S, Pinault M, Pfefferle LD, Elimelech M. Single-walled carbon nanotubes exhibit strong antimicrobial activity. Langmuir. 2007;23:8670-3. PMID: 17658863 DOI: $10.1021 /$ la701067r

Krishnaraj RN, Chandran S, Pal P, Berchmans S. Investigations on the antiretroviral activity of carbon nanotubes using computational molecular approach. Comb Chem High Throughput Screen. 2014;17:531-5. PMID: 24433396 DOI: $10.2174 / 1386207317666140116110558$

Liu $\mathrm{H}$, Zhang $\mathrm{L}$, Yan $\mathrm{M}$, Yu J. Carbon nanostructures in biology and medicine. J Mater Chem B. 2017;5:6437-50. PMID: 32264410 DOI: 10.1039/C7TB00891K

Liu Y, Chen C. Effect on Reproductive System of Carbon Nanomaterials. In: Chen $\mathrm{C}$, Wang $\mathrm{H}$, eds. Biomedical Applications and Toxicology of Carbon Nanomaterials. Hoboken: John Wiley \& Sons; 2016. p. 333-56.

Maleki Dizaj S, Mennati A, Jafari S, Khezri K, Adibkia K. Antimicrobial activity of carbon-based nanoparticles. Adv Pharm Bull. 2015;5:19-23. PMID: 25789215 DOI: 10.5681/apb.2015.003

Mukhin IS, Fadeev IV, Zhukov MV, Dubrovskii VG, Golubok AO. Framed carbon nanostructures: synthesis and applications in functional SPM tips. Ultramicroscopy. 2015;148:151-7. PMID: 25461592 DOI: 10.1016/j.ultramic. 2014.10.008

Patel DK, Kim HB, Dutta SD, Ganguly K, Lim KT. Carbon Nanotubes-Based Nanomaterials and Their Agricultural and Biotechnological Applications. Materials (Basel). 2020;13:1679. PMID: 32260227 DOI: 10.3390/ ma13071679

Ramal-Sanchez M, Valbonetti L, Tsikis G, Dubuisson F, Blache MC, Labas V, Druart X, Fontana A, Mermillod P, Barboni B, Saint-Dizier M, Bernabo N. Graphene Oxide: A glimmer of hope for assisted reproductive technology. Carbon. 2019;150:518-30. DOI: 10.1016/j.carbon.2019.05.055

Saliev T. The Advances in Biomedical Applications of Carbon Nanotubes. J Carbon Res. 2019;5:29. DOI: 10.3390/ c5020029

Vasyukova I, Gusev A, Tkachev A. Reproductive toxicity of carbon nanomaterials: a review. IOP Conf Ser Mater Sci Eng. 2015;98:012001. DOI: 10.1088/1757899X/98/1/012001
Xu C, Liu Q, Liu H, Zhang C, Shao W, Gu A. Toxicological assessment of multi-walled carbon nanotubes in vitro: potential mitochondria effects on male reproductive cells. Oncotarget. 2016;7:39270-8. PMID: 27248475 DOI: 10.18632/oncotarget. 9689

Zahid M, Kim B, Hussain R, Amin R, Park SH. DNA nanotechnology: a future perspective. Nanoscale Res Lett. 2013;8:119. PMID: 23497147 DOI: 10.1186/1556-276X8-119

Zare-Zardini H, Amiri A, Shanbedi M, Memarpoor-Yazdi M, Asoodeh A. Studying of antifungal activity of functionalized multiwalled carbon nanotubes by microwave-assisted technique. Surf Interface Anal. 2013;45:751-5.

Zare-Zardini H, Amiri A, Shanbedi M, Taheri-Kafrani A, Sadri Z, Ghanizadeh F, Neamatzadeh H, Sheikhpour R, Keyvani Boroujeni F, Masoumi Dehshiri R, Hashemi A, Aminorroaya MM, Dehgahnzadeh MR, Shahriari Sh. Nanotechnology and Pediatric Cancer: Prevention, Diagnosis and Treatment. Iran J Ped Hematol Oncol. 2015;5:233-48. PMID: 26985357

Zare-Zardini $H$, Ferdowsian $F$, Soltaninejad $H$, Ghorani Azam A, Soleymani S, Zare-Shehneh M, Mofidi M, Rafati R, Ebrahimi L. Application of Nanotechnology in Biomedicine: A Major Focus on Cancer Therapy. J Nano Res. 2016;25:55-66.

Zare-Zardini H, Taheri-Kafrani A, Ordooei M, Amiri A, Karimi-Zarchi M. Evaluation of toxicity of functionalized graphene oxide with ginsenoside Rh2, lysine and arginine on blood cancer cells (K562), red blood cells, blood coagulation and cardiovascular tissue: In vitro and in vivo studies. J Taiwan Inst Chem Eng. 2018a;93:70-8. DOI: 10.1016/j.jtice.2018.08.010

Zare-Zardini H, Taheri-Kafrani A, Amiri A, Bordbar AK. New generation of drug delivery systems based on ginsenoside Rh2-, Lysine- and Arginine-treated highly porous graphene for improving anticancer activity. Sci Rep. 2018b;8:586. PMID: 29330486 DOI: 10.1038/s41598-017-18938-y

Zare-Zardini $H$, Shanbedi $M$, Soltaninejad $H$, Mohammadzadeh M, Amiri A, Hamidieh AA, Ferdosian F, Alemi A, Hosseinkhani S, Fesahat F, Astani A. The Effect of Temperature and Acidity on Antimicrobial Activities of Pristine MWCNTs and MWCNTs-Arg. Int J Nanosci Nanotechnol. 2020;12:127-36. 